\title{
Novel Application of Point-of-Care Ocular Ultrasound in a Left Central Retinal Artery Occlusion
}

\author{
Rachel E. Bridwell ${ }^{1}$, Jesse Wray ${ }^{1}$, Joshua Oliver $^{1}$, Amber Cibrario ${ }^{1}$, Melissa Myers ${ }^{1}$ \\ 1. Emergency Medicine, Brooke Army Medical Center, Fort Sam Houston, USA
}

Corresponding author: Rachel E. Bridwell, r.e.bridwell@gmail.com

\begin{abstract}
Central retinal artery occlusion represents a vision-threatening entity in those presenting with monocular painless vision loss, especially in the elderly and those with cardiovascular comorbidities. While confirmation of this diagnosis requires consultation with an ophthalmologist, prompt recognition is the crucial action of the emergency physician to help reverse retinal ischemia and save vision. Here we describe the case of a central retinal artery occlusion identified on point-of-care ocular ultrasound and confirmed by fluorescein angiography.
\end{abstract}

Categories: Emergency Medicine, Ophthalmology

Keywords: central retinal artery occlusion, ultrasound

\section{Introduction}

Central retinal artery occlusion (CRAO) is a rare but vision-threatening event occurring in 1-2 in 100,000 patients, though this increases with age, occurring 10 times more commonly in patients over 80 years of age [1-3]. Direct fundoscopy, a mainstay of initial CRAO diagnosis until fluorescein angiography can be performed, is not a central skill for the emergency physician, which is the mainstay of initial CRAO diagnosis. There is a paucity of evidence in the literature in which point-of-care ultrasound (POCUS) has been used to diagnose CRAO. We present the case below with painless monocular vision loss of a thrombus identified on emergency POCUS.

\section{Case Presentation}

A 63-year-old woman with a history of coronary artery disease, diabetes mellitus, and atrial fibrillation not on anticoagulation presented to the emergency department (ED) with acute-onset persistent painless vision loss in her left eye two hours prior to arrival. Review of systems was notable only for a left-sided temporal headache.

Received 03/23/2020 Review began 03/27/2020 Review ended 03/30/2020 Published 04/02/2020

(c) Copyright 2020 Bridwell et al. This is an open access article distributed under the terms of the Creative Commons Attribution License CC-BY 4.0, which permits unrestricted use, distribution, and reproduction in any medium, provided the original author and source are credited.
On arrival, the vital signs were within normal limits. The physical examination was notable for an inability to perceive light or movement in the left eye with an afferent pupillary defect. POCUS demonstrated a hyperechoic circular structure in the center of the left optic nerve (Figure 1). A computed tomography (CT) of the head and CT angiography of the neck were obtained, which demonstrated atherosclerotic disease of the bilateral carotid, temporal, and ophthalmic arteries, but there was no evidence of acute intracranial ischemia or hemorrhage. She was placed on $100 \%$ fraction of inspired oxygen due to a concern for CRAO. Both neurology and ophthalmology providers were consulted, who recommended hyperbaric oxygen therapy. Because giant cell arteritis (GCA) was an alternative possible etiology of the presentation, she was empirically started on high-dose corticosteroids. A subsequent temporal artery biopsy found no evidence of vasculitis. She was admitted to the medical ward and underwent a hyperbaric oxygen protocol without improvement in her visual deficit. CRAO was observed on direct fundoscopy and confirmed on fluorescein angiography (Figure 2). Additional cerebrovascular accident (CVA) workup to include transthoracic echocardiography and magnetic resonance imaging (MRI) with angiography demonstrated no patent foramen ovale or acute findings. After discharge, an outpatient ophthalmology follow-up demonstrated no light perception in the left eye. 


\section{Cureus}

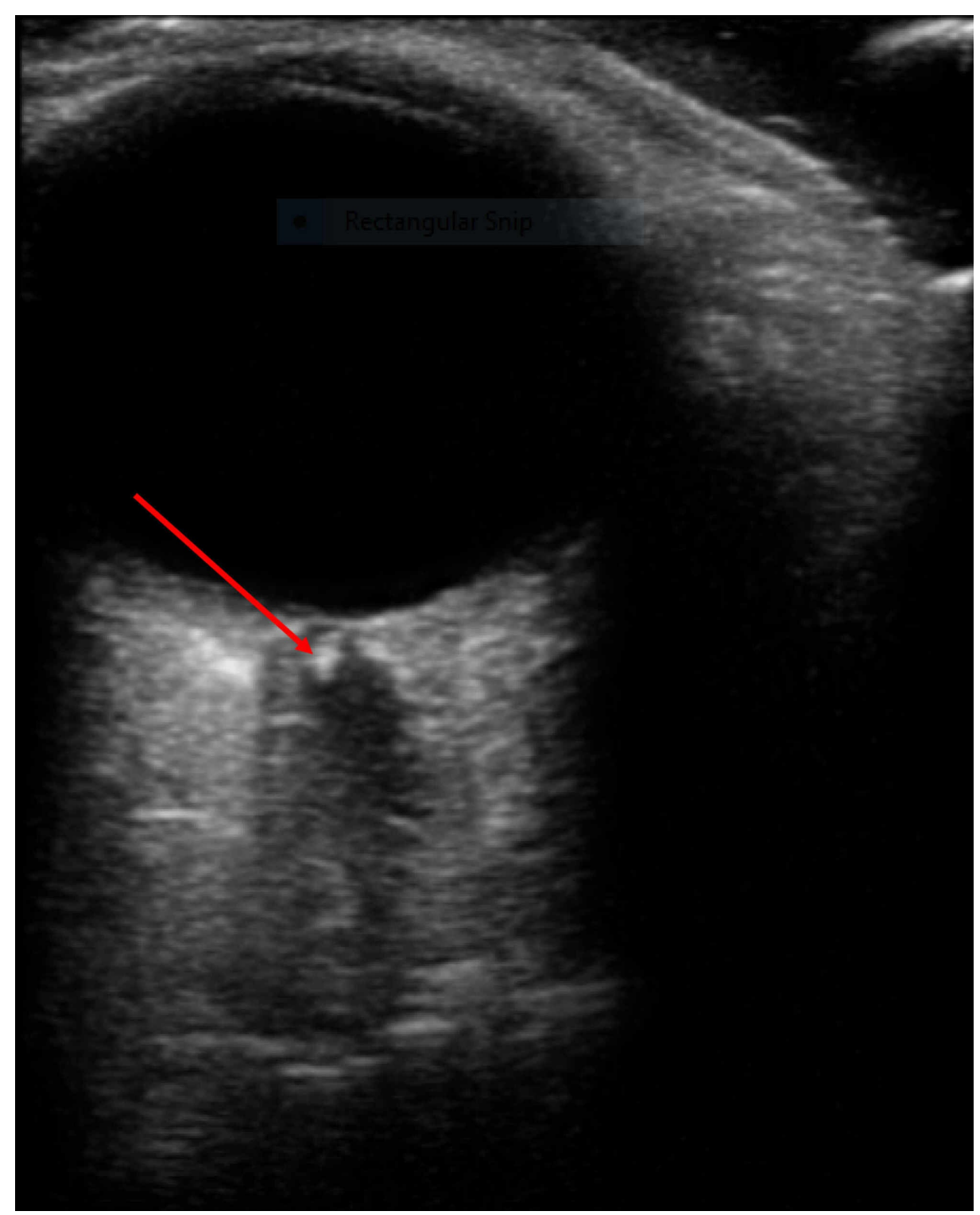

FIGURE 1: Transverse linear array ultrasound of the left eye demonstrating central retinal artery occlusion (red arrow).

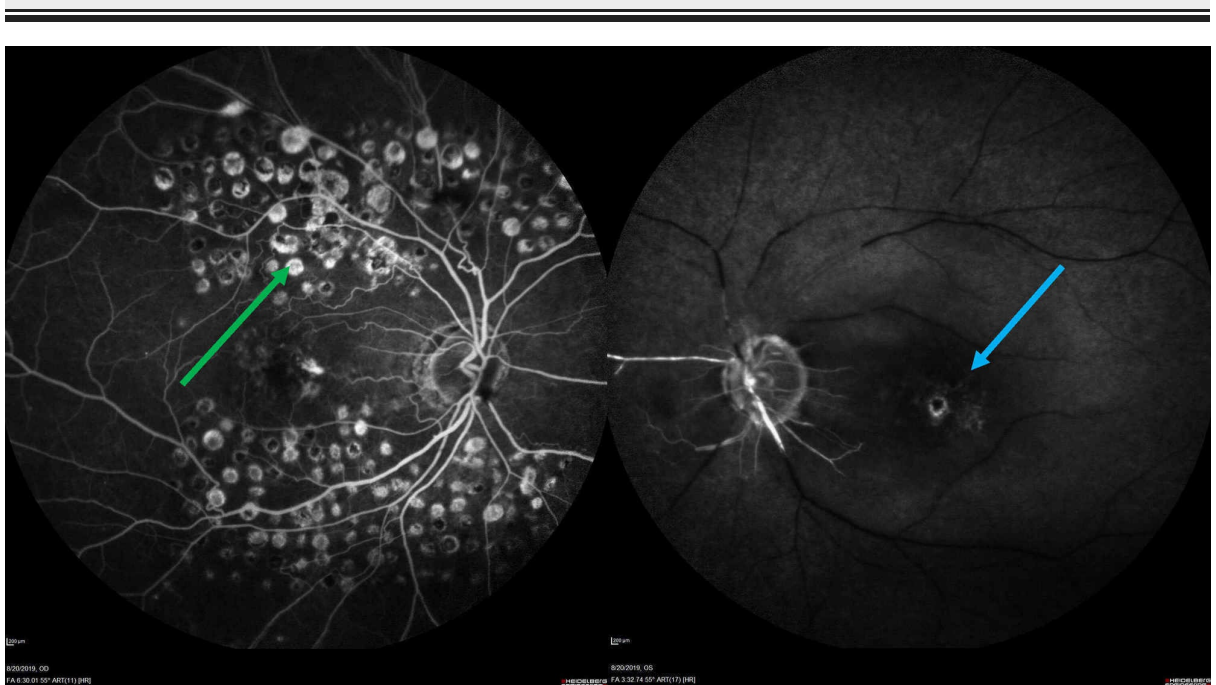

FIGURE 2: Fluorescein angiography demonstrating central retinal artery 


\section{Discussion}

CRAO represents a portion of patients presenting with monocular painless vision loss, in which retinal ischemic time determines vision. While rare, its incidence increases exponentially until the eighth decade of life and is associated with coronary artery disease, diabetes mellitus, CVA, transient ischemic attack, hyperlipidemia, smoking, and hypercoagulable states, with embolism representing as the most common etiology $[2,3]$. Currently, the mainstay of diagnostic workup in the ED relies heavily on direct fundoscopy, which is a dying art for the emergency physician. Traditional diagnostic criteria include the presence of "box-carring" of the retinal vessels in conjunction with retinal opacity with a cherry red spot [2]. After establishing these examination findings, the diagnosis is traditionally confirmed with a fluorescein angiography [1]. There are only a few limited studies describing the use of ultrasound to identify CRAO [4,5]. There are no definitive sensitivities for POCUS, though $31 \%$ of CRAOs demonstrated retrobulbar hyperechoic foci on orbital color Doppler imaging, suggesting the possible utility of ultrasound [4]. The presence of a visible thrombus such as the one described may expedite the workup; primate models and human observational studies have demonstrated that the duration of retinal ischemic time significantly determines visual damage $[1,6,7]$.

In ED management, any clinical presentation suggesting GCA should initiate inflammatory markers and prompt administration of steroids. In a study of retinal artery occlusions, 12 cases experienced CRAO secondary to GCA [2]. In this small subset, GCA causes posterior ciliary artery and cilioretinal artery occlusion [8]. CRAO also portends a higher risk of subsequent myocardial infarction or cerebral infarction, especially within the first week following a CRAO [1]. Emergency physicians should not only recognize the vision-threatening aspect of this disease but also recognize it as a marker of poor cardiovascular status with an increased risk of other cardiovascular morbidity and mortality $[9,10]$.

Workup in the ED should include a parallel CVA workup with an MRI with diffusion-weighted imaging if possible, as CRAO is a stroke equivalent with retinal tissue ischemia [11]. The treatment of CRAO presents the ultimate challenge as a few successful treatments have been described in data. Ocular massage, laser embolectomy, minimization of intra-ocular pressure, hyperbaric oxygen, and intra-arterial thrombolysis have not demonstrated improvement in vision [12-16]. Given the high risk of both cardiovascular complications and additional hypercoagulability workup, patients should be admitted [2].

\section{Conclusions}

CRAO is a rare but vision-threatening diagnosis in those presenting with painless monocular vision loss, more common in the elderly and those with cardiovascular medical comorbidities. Prompt recognition and diagnosis is paramount in reversing the ongoing retinal vascular ischemia. Previously described in only a few cases, POCUS may identify distal thrombi and expedite this time-sensitive workup.

\section{Additional Information \\ Disclosures}

Human subjects: Consent was obtained by all participants in this study. Conflicts of interest: In compliance with the ICMJE uniform disclosure form, all authors declare the following: Payment/services info: All authors have declared that no financial support was received from any organization for the submitted work. Financial relationships: All authors have declared that they have no financial relationships at present or within the previous three years with any organizations that might have an interest in the submitted work. Other relationships: All authors have declared that there are no other relationships or activities that could appear to have influenced the submitted work.

\section{Acknowledgements}

The view(s) expressed herein are those of the author(s) and do not reflect the official policy or position of Brooke Army Medical Center, the U.S. Army Medical Department, the U.S. Army Office of the Surgeon General, the Department of the Army, the Department of the Air Force, the Department of Defense, or the U.S. Government.

\section{References}

1. Dattilo M, Biousse V, Newman NJ: Update on the management of central retinal artery occlusion . Neurol Clin. 2017, 35:83-100. 10.1016/j.ncl.2016.08.013

2. Hayreh SS, Podhajsky PA, Zimmerman MB: Retinal artery occlusion. associated systemic and ophthalmic abnormalities. Ophthalmology. 2009, 116:1928-1936. 10.1016/j.ophtha.2009.03.006 
3. Park SJ, Choi NK, Seo KH, Park KH, Woo SJ: Nationwide incidence of clinically diagnosed central retinal artery occlusion in Korea, 2008 to 2011. Ophthalmology. 2014, 121:1933-1938.

10.1016/j.ophtha.2014.04.029

4. Stoner-Duncan B, Morris SC: Early identification of central retinal artery occlusion using point-of-care ultrasound. Clin Prac Cases Emerg Med. 2019, 3:13-15. Accessed: April 2, 2020:

doi:10.5811/cpcem.2018.11.39406

5. Riccardi A, Siniscalchi C, Lerza R: Embolic central retinal artery occlusion detected with point-of-care ultrasonography in the emergency department. J Emerg Med. 2016, 4:183-185.

https://doi.org/10.1016/j.jemermed.2015.12.022

6. Hayreh SS, Jonas JB: Optic disk and retinal nerve fiber layer damage after transient central retinal artery occlusion: an experimental study in rhesus monkeys. Am J Ophthalmol. 2000, 129:786-795. 10.1016/S00029394(00)00384-6

7. Hayreh SS, Zimmerman MB, Kimura A, Sanon A: Central retinal artery occlusion. Retinal survival time . Exp Eye Res. 2004, 78:723-736. 10.1016/S0014-4835(03)00214-8

8. Hayreh SS, Podhajsky PA, Zimmerman B: Ocular manifestations of giant cell arteritis. Am J Ophthalmol. 1998, 125:509-520. 10.1016/S0002-9394(99)80192-5

9. Callizo J, Feltgen N, Pantenburg S, et al.: Cardiovascular risk factors in central retinal artery occlusion: results of a prospective and standardized medical examination. Ophthalmology. 2015, 122:1881-1888. 10.1016/j.ophtha.2015.05.044

10. Chang YS, Jan RL, Weng SF, et al.: Retinal artery occlusion and the 3-year risk of stroke in Taiwan: A nationwide population-based study. Am J Ophthalmol. 2012, 154:645-652. 10.1016/j.ajo.2012.03.046

11. Janis LS, Connors JJ, George MG, et al.: An updated definition of stroke for the 21st century: a statement for healthcare professionals From the American Heart Association/American Stroke Association. Stroke. 2013, 44:2064-2089. 10.1161/str.0b013e318296aeca

12. Rudkin AK, Lee AW, Aldrich E, Miller NR, Chen CS: Clinical characteristics and outcome of current standard management of central retinal artery occlusion. Clin Exp Ophthalmol. 2010, 38:496-501. 10.1111/j.14429071.2010.02280.x

13. Reynard M, Hanscom TA: Neodymium:yttrium-aluminum-garnet laser arteriotomy with embolectomy for central retinal artery occlusion. Am J Ophthalmol. 2004, 137:196-198. 10.1016/S0002-9394(03)00817-1

14. Rumelt S, Dorenboim Y, Rehany U: Aggressive systematic treatment for central retinal artery occlusion. Am J Ophthalmol. 1999, 128:733-738. 10.1016/S0002-9394(99)00359-1

15. Menzel-Severing J, Siekmann U, Weinberger A, Roessler G, Walter P, Mazinani B: Early hyperbaric oxygen treatment for nonarteritic central retinal artery obstruction. Am J Ophthalmol. 2012, 153:454-459. 10.1016/j.ajo.2011.08.009

16. Schumacher M, Schmidt D, Jurklies B, et al.: Central retinal artery occlusion: Local intra-arterial fibrinolysis versus conservative treatment, a multicenter randomized trial. Ophthalmology. 2010, 117:1367-1375. 10.1016/j.ophtha.2010.03.061 